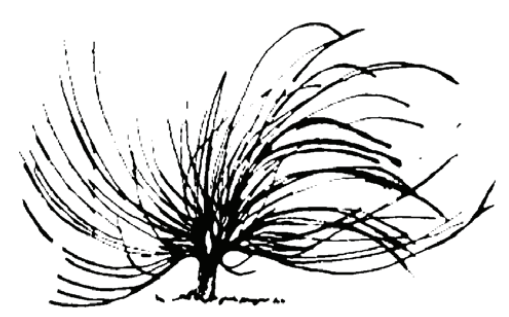

\title{
Métodos para la Enseñanza de Idiomas: No Hay Nada Nuevo Bajo el Sol, ¿O Sí?
}

\author{
Juan Pablo Zúñiga Vargas ${ }^{1}$ \\ Universidad de Costa Rica \\ San José, Costa Rica \\ juanpablo.zunigavargas@ucr.ac.cr
}

\begin{abstract}
Resumen
Este ensayo tiene como objetivo problematizar la posible dirección que seguirá el campo de la enseñanza de idiomas en el siglo XXI. Para tal fin, se describirán nueve parámetros metodológicos para la conceptualización de todo método para la enseñanza de idiomas, se reflexionará sobre la imposibilidad de designar el mejor método para la enseñanza de idiomas, se discutirá el concepto de la condición postmétodos y sus diez macro- estrategias para la enseñanza de idiomas junto con los desafíos para su puesta en práctica, se hará referencia a una propuesta minimalista para la enseñanza de idiomas y finalmente se analizará el fenómeno actual de la práctica definida por el libro de texto.
\end{abstract}

Palabras clave: métodos, enseñanza, idiomas, pedagogía, condición postmétodos, futuro

\section{(ब) $\Theta \Theta$}

Recibido: 23 de octubre de 2015-Aprobado: 4 de diciembre de 2015

1 Profesor de inglés para otras carreras, Universidad de Costa Rica. Profesor de pedagogía e investigación educativa para la enseñanza del inglés, Universidad Nacional, Costa Rica. Magíster en la Enseñanza del Inglés como Lengua Extranjera y Bachiller en Inglés, ambos títulos obtenidos en la Universidad de Costa Rica. 


\begin{abstract}
This essay intends to problematize the possible direction that the field of language teaching will follow in the XXI century. For this purpose, nine methodological parameters for conceptualizing every language teaching method will be described, the impossibility of designating the best method will be considered, the concept of the post method condition and its ten macro strategies for language teaching will be discussed along with the challenges for its implementation, a minimalist proposal for language teaching will be referred to, and the current phenomenon of textbook-defined practice will be analyzed.
\end{abstract}

Keywords: languages, teaching, methods, pedagogy, postmethod condition, future

\title{
Introducción
}

El presente ensayo tiene como objetivo primordial problematizar la posible dirección que ha de seguir la disciplina de la enseñanza de idiomas en el siglo XXI, así como describir qué puede (no) ser novedoso en este campo del conocimiento. Antes de iniciar propiamente con la discusión, es importante hacer una aclaración básica: en este ensayo, se utilizará la palabra "método" de manera general para referirse tanto a los métodos como a los enfoques para la enseñanza de idiomas, sin ánimo alguno de causar polémica o debates en cuanto a las diferencias epistemológicas entre estos dos conceptos.

Hecha esta aclaración inicial, se describirá seguidamente la manera en la cual se estructurará este ensayo. Primero se analizarán una serie de dimensiones para la enseñanza de idiomas, las cuales son aplicables a todos los métodos existentes y por proponer en el campo de la enseñanza de idiomas. Posteriormente, se reflexionará sobre la dificultad o imposibilidad de designar el mejor método para la enseñanza de idiomas. La condición post métodos y algunas recomendaciones metodológicas contemporáneas también se analizarán para tratar de dilucidar los posibles caminos a seguir en la enseñanza de idiomas del siglo XXI. Se describirán además los principales retos a enfrentar en este paradigma emergente, junto con una propuesta minimalista para la enseñanza 
de idiomas y el fenómeno actual de la práctica definida por el libro de texto. Se espera, por lo tanto, que las reflexiones y conclusiones alcanzadas en este escrito sirvan como insumo a docentes de idiomas experimentados y principiantes que deseen (re)examinar su práctica actual desde un punto de vista crítico y con miras a su crecimiento profesional.

\section{Las Nueve Dimensiones de los Métodos para la Enseñanza de Idiomas}

En todo programa de formación para docentes de idiomas, es común que al menos uno de los cursos de esa malla curricular tenga como temática central los métodos para la enseñanza de idiomas. El objetivo de este curso, sin embargo, no siempre es tan claro como se argumentará inmediatamente. En algunos casos, podría tener como meta el resaltar la importancia de que los docentes de idiomas en formación conozcan el desarrollo que ha tenido la disciplina o que, al estudiar diferentes métodos, puedan algún día enriquecer su práctica profesional al combinar elementos (estrategias, técnicas u otros) de diversos métodos. Sin embargo, tal estudio teórico puede que no sea más que un mero ejercicio intelectual con pocas aplicaciones pragmáticas en la práctica profesional de los docentes de idiomas.

Es común también que, los libros o antologías utilizados en estos cursos tengan una organización cronológica con respecto al período de origen de los métodos que se vayan a estudiar. Tal organización temática parece sugerir que la disciplina de enseñanza de idiomas alguna vez estuvo sumida en las sombras y es hasta épocas recientes que se camina hacia la luz. No obstante, esta idea podría estar equivocada, como lo sugieren Kumaravadivelu (2003), al considerar que, existe bastante superposición teórica en once métodos descritos en dos de los libros más comúnmente empleados en cursos sobre enseñanza de idiomas y Thornbury (2010), quien propone nueve dimensiones para la conceptualización de todos los métodos para la enseñanza de idiomas. Según argumenta Thornbury (2010) de manera certera, el desarrollo de la enseñanza de idiomas no ha sido lineal, sino más bien ha sido una espiral, en la cual las mismas dimensiones se han utilizado de manera diferente para dar sentido a los diferentes métodos para la enseñanza de idiomas. En este momento, es importante aclarar que, estas dimensiones no deben verse como dicotomías o conjuntos de nociones opuestas, 
sino como espectros de posibilidades para el ejercicio de la enseñanza de idiomas. Por consiguiente, cada método estaría orientado más hacia la derecha o hacia la izquierda en cada uno de estos espectros según su conceptualización específica. Cada una de estas dimensiones se describirá a continuación.

\section{Dimensión 1: El Lenguaje}

Todo método para la enseñanza de idiomas presupone una visión sobre la naturaleza del lenguaje (ver figura 1). El lenguaje puede ser visto como un conjunto de reglas (gramaticales, morfológicas, fonológicas, entre otras) y su aprendizaje y uso consistirían en el dominio de tales reglas. Por otro lado, el lenguaje puede ser visto más en términos de su función. En este sentido, se infiere que el lenguaje puede ser visto más como un fenómeno social, en el cual aprender y utilizar un idioma estarían supeditados a su fin último: la comunicación.

Figura 1. La Naturaleza del Lenguaje

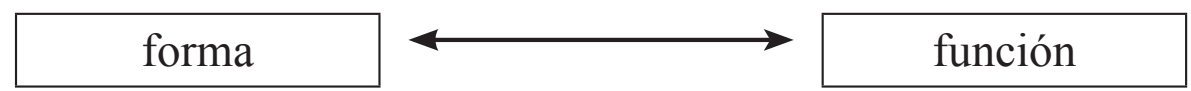

Nota: Adaptado de Thornbury (2010)

\section{Dimensión 2: El Aprendizaje}

Los diversos métodos para la enseñanza de idiomas toman una perspectiva cognoscitiva o vivencial con respecto al concepto del aprendizaje (ver figura 2). El aprendizaje de un nuevo idioma entonces puede verse como un proceso meramente analítico, de descubrir reglas y principios, ya sea de manera inductiva o deductiva. No obstante, aprender un idioma en otros métodos es algo más vivencial, hay que experimentar el lenguaje, hay que vivirlo para que el aprendizaje ocurra.

Figura 2. La Naturaleza del Aprendizaje

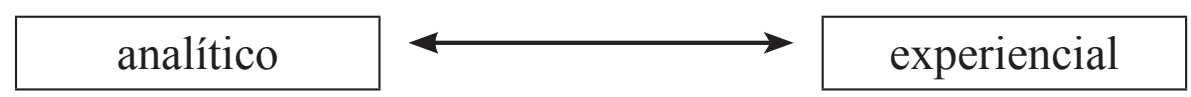

Nota: Adaptado de Thornbury (2010) 


\section{Dimensión 3: El Fin Último de Aprender un Idioma}

Hay métodos que ven el propósito de aprender un idioma como un ejercicio mental o de crecimiento personal. El haber aprendido un idioma significa ser capaz de usarlo con gramática y pronunciación impecables. Otros métodos, sin embargo, abogan por desarrollar la razón primordial de ser de un lenguaje: la necesidad de comunicarse con otros (ver figura 3). Sin esta necesidad básica, ningún lenguaje existiría. Esta visión, en consecuencia, afectará en gran medida las actividades que se lleven a cabo en el aula.

Figura 3. Meta de Aprender un Idioma

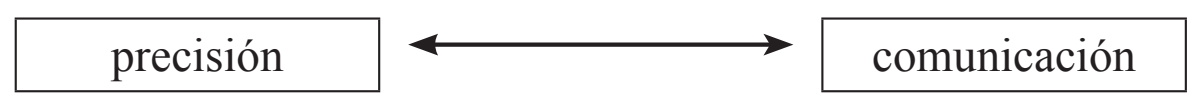

Nota: Adaptado de Thornbury (2010)

\section{Dimensión 4: Organización de Un Curso de Idiomas}

A nivel de la organización de contenidos en un curso de idiomas, en general existen las alternativas de estructurar un curso con una visión integral o específica del lenguaje (ver figura 4). Un curso integrado ve el lenguaje más como un sistema compuesto por diferentes partes, las cuales son todas importantes para su aprendizaje y eventual adquisición. Hay otros cursos en los cuales es necesario o incluso más práctico separar el lenguaje en sus macrodestrezas (escucha, habla, lectura y escritura) o en sus microdestrezas (gramática y pronunciación, por ejemplo) para que el estudiante pueda crecer lingüísticamente al concentrarse específicamente en cursos dedicados principalmente a una destreza.

\section{Figura 4. El Principio de Organización de un Curso de Idiomas}

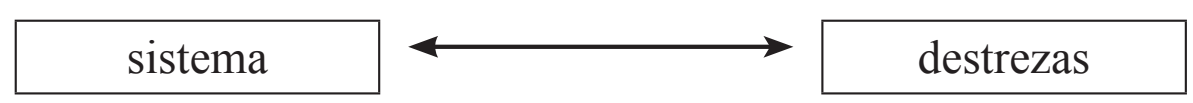

Nota: Adaptado de Thornbury (2010) 


\section{Dimensión 5: El Currículum}

En el ámbito curricular, un curso de idiomas puede ser independiente o ser parte de una estructura mayor (ver figura 5). En un curso de idiomas que esté segregado del resto del currículum, se estudian temas de interés general cuyo fin primordial es el aprendizaje del idioma. Por otro lado, si un curso de idiomas está integrado curricularmente, en él se puede aprender sobre otras materias (historia, ciencias, matemáticas, por citar algunas). Entonces el propósito de un curso de idiomas de tal índole es tanto aprender el idioma como crecer personal y profesionalmente al abordar materias variadas. Este modelo de integración curricular es el que se puede encontrar en los centros educativos bilingües.

Figura 5. El Currículum para el Aprendizaje de Idiomas

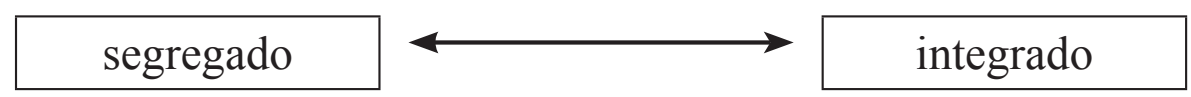

Nota: Adaptado de Thornbury (2010)

\section{Dimensión 6: El Aprendiz y los Materiales de Idiomas}

No todos los métodos para la enseñanza de idiomas propuestos han brindado una visión explícita sobre cuál rol deberían desempeñar el aprendiz de idiomas y los materiales usados en un curso. Sin embargo, es posible identificar dos papeles principales en este respecto (ver figura 6). Algunos métodos han concebido a los estudiantes como seres eminentemente cognoscitivos, cuyos retos y tareas para aprender un nuevo idioma son meramente mentales. Por lo tanto, los materiales que se desarrollen en un curso con esta perspectiva han de asistir a los estudiantes en estos ejercicios intelectuales. Otros métodos ven a los estudiantes como seres humanos integrales provistos de una esfera afectiva, la cual debe ser estimulada para lograr un aprendizaje significativo, por lo que los materiales que se diseñen dentro de este paradigma deben contribuir de una u otra manera a que los estudiantes moderen su filtro afectivo. 
Figura 6. Rol del Aprendiz y los Materiales de Idiomas

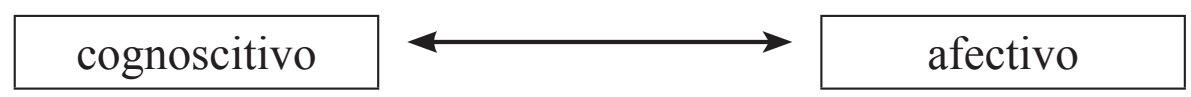

Nota: Adaptado de Thornbury (2010)

\section{Dimensión 7: EI Docente de Idiomas}

El papel que desempeña el docente de idiomas se ha conceptualizado desde dos ámbitos principales, el transmisivo y el dialógico (ver figura 7). Tradicionalmente se ha visto al docente como una figura de autoridad, casi incuestionable, cuyo deber es transmitir conocimientos unidireccionalmente. Visiones más contemporáneas, sin embargo, han propuesto que el docente puede tener un rol más dialógico y los procesos de enseñanza-aprendizaje ser más horizontales. Dicho de otro modo, el docente no necesita saberlo todo; el docente es humano y tanto docentes como estudiantes pueden aprender uno del otro.

Figura 7. Rol del Docente de Idiomas

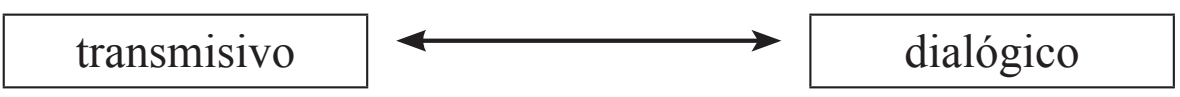

Nota: Adaptado de Thornbury (2010)

\section{Dimensión 8: El Proceso el Aprendizaje de Idiomas}

En procesos de enseñanza y aprendizaje de idiomas, se han empleado dos paradigmas principales. Ciertos métodos abogan por enseñarle a los estudiantes reglas del idioma de manera explícita para que posteriormente se pongan en práctica por medio de la redacción de oraciones o la pronunciación de sonidos o fonemas específicos, mientras que otros métodos sugieren que a los estudiantes se les guíe en un proceso de autodescubrimiento de reglas y principios lingüísticos (ver figura 8). En relación con esta dimensión, la experiencia parece sugerir que la inducción es más efectiva. No obstante, la deducción también debería estimularse en clase, ya que no se puede aprender todo en un aula y en la vida real será necesario enfrentarse con lo desconocido. 
Figura 8. Paradigma del Proceso del Aprendizaje de Idiomas

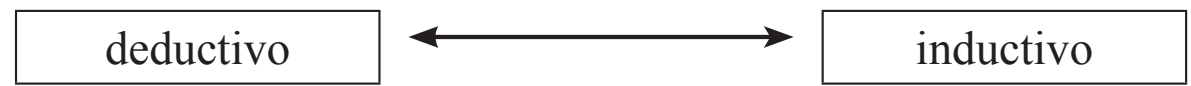

Nota: Adaptado de Thornbury (2010)

\section{Dimensión 9: Medio de Instrucción para la Enseñanza de Idiomas}

Diferentes métodos han sugerido cuál debería ser el idioma predominante en las sesiones de clase, sea el idioma nativo o el idioma meta (ver figura 9). Por eso, métodos como el de Gramática y Traducción emplean la traducción como la principal técnica de enseñanza. Por otro lado, el Método Directo prohibió la traducción y se centró en el uso exclusivo de la lengua meta. Cualquiera de estos extremos supone fortalezas y debilidades, por lo que el docente de idiomas debe encontrar los momentos más pertinentes para moverse a la izquierda o a la derecha de este espectro.

\section{Figura 9. Medio de Instrucción Primordial en la Clase de Idiomas}

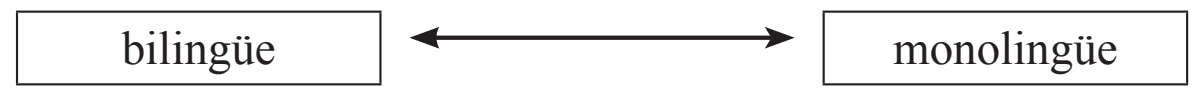

Nota: Adaptado de Thornbury (2010)

Como se dijo inicialmente, ningún método para la enseñanza de idiomas (existente o por venir) es necesariamente nuevo o auténtico, sino más bien una permutación diferente de todas (o la gran mayoría) de las nueve dimensiones que propone Thornbury (2010). Thornbury (2010) sugiere también que estas nueve dimensiones pueden tener implicaciones metodológicas a la hora de planear lecciones para un curso de idiomas. Los diferentes espectros de posibilidades presentes en cada una de las dimensiones se pueden adaptar a conveniencia de un docente de idiomas. En este sentido, podemos sugerir una comparación simple: Podemos ver todas las dimensiones como las bandas para ajustar el sonido en un aparato reproductor de audio. Según las necesidades estudiantiles, contextuales y didácticas con las que tenga que lidiar un docente de idiomas, la configuración de sus lecciones puede variar de derecha a izquierda y viceversa en cada una de las dimensiones para sacar el mejor provecho de las experiencias de enseñanza y aprendizaje 
en un curso de idiomas. En consecuencia, si ciertos elementos explícitos e implícitos en los métodos para la enseñanza de idiomas lleva a la conclusión de que ningún método es verdaderamente auténtico, pensar en cuál podría ser el mejor es un problema aún mayor, tal y como se argumentará en los siguientes párrafos.

\section{EI Dilema de Designar a un Método como el Mejor}

En cursos de formación docente en los cuales se estudien diferentes métodos para la enseñanza de idiomas, es común que una pregunta recurrente surja: “¿Profesor, cuál es el mejor método?” Esta es una pregunta complicada, ya que puede que no tenga respuesta o puede que tenga múltiples respuestas. Basta con considerar el siguiente escenario hipotético: ¿En cuál situación se obtendrían mejores resultados, en un curso en el cual se utilice el "mejor" método puesto en práctica por un docente poco hábil o en un curso en el cual se utilice un método "malo" puesto en práctica por un docente apasionado? Desde el siglo pasado, Prabhu (1990) consideró que no existe una respuesta para esta interrogante por diversas razones que se explicarán seguidamente.

\section{El Contexto Determina la Mejor Metodología}

En cada proceso de enseñanza y aprendizaje, el contexto socio-económico y socio-educativo va a ser siempre distinto y cada método para la enseñanza de idiomas fue concebido para ser puesto en práctica en un contexto genérico e ideal. La combinación de factores motivacionales, experienciales, actitudinales, aptitudinales, económicos, filosóficos, curriculares, culturales (por mencionar solo algunos) afectará de manera directa, la forma en la cual se enseña y se aprende. Quiere decir entonces que, la escogencia de métodos (o metodologías) de enseñanza debe realizarse de manera crítica y flexible con el fin de responder a las diversas características que moldean cada contexto educativo. De este modo, se podrá elegir el mejor modo de enseñar un idioma. No se puede pensar de una manera reduccionista y esperar que un mismo método para la enseñanza de idiomas funcione bien en cualquier contexto educativo. 


\section{Cada Método Tiene su Validez}

Diversos métodos han emergido y desaparecido en la historia de la enseñanza de idiomas y algunos han sido más populares o más efectivos que otros. No obstante, una característica común a todos los métodos es la promoción del aprendizaje; cualquiera ha de aprender algo con cualquier método o, dicho de otro modo, ningún método habría sido documentado y descrito de no proveer algún beneficio. Por esta razón, se ha creído que si es posible identificar lo mejor de cada método, todas estas "buenas" características deberían poder combinarse para crear una metodología superior. Sin embargo, realizar tal mezcla puede tener sus inconvenientes.

\section{El Eclecticismo Puede Ser Peligroso}

La noción del eclecticismo ha estado en boga por mucho tiempo y muchos parecen aceptarla sin mayor objeción. Incluso en los cursos de métodos para la enseñanza de idiomas se cree que, al enseñarle a los futuros docentes los principales métodos que han surgido en la historia de la disciplina, ellos integrarán elementos de diferentes métodos en su práctica profesional. En este punto cabe preguntarse si esto realmente es posible o deseable. Si ciertos métodos pueden funcionar mejor en ciertos contextos. ¿Cómo se puede identificar lo mejor que pueda tener cada uno de estos métodos? Y si esta identificación de lo que es mejor fuera posible, ¿cómo se podría determinar que la mezcla ecléctica resultante realmente es compatible o útil? También el eclecticismo podría convertirse en un estandarte acrítico para justificar cualquier tipo de práctica en un salón de clases, sea esta beneficiosa o no y al ser cuestionado un docente sobre las actividades que realiza, este puede simplemente decir "mi metodología es ecléctica" y quienes escucharan esta respuesta probablemente no podrían emitir juicios claros al respecto.

\section{No Hay Criterios Claros para Designar al Mejor Método}

El poder medir o valorar objetivamente los resultados de aplicar cualquier método en comparación con algún otro constituye un obstáculo quizás infranqueable. Por ejemplo, se podría realizar un experimento en el cual dos o más grupos de estudiantes fueran instruidos 
con métodos diferentes para luego ser evaluados con los mismos exámenes o instrumentos. Se podría creer ingenuamente que aquel grupo con el mejor desempeño fue el que tuvo el mejor método, pero como se mencionó anteriormente, el contexto influiría grandemente en la efectividad. Incluso si fuera posible enseñar a un grupo de estudiantes con un método y luego hacer que olvidaran por completo esta experiencia para utilizar uno diferente y comparar los resultados, siempre habría una duda razonable de que las puestas en práctica de estos métodos no fueran realmente distintas. Como Thornbury (2010) ya ha dicho, los diferentes métodos para la enseñanza de idiomas pueden verse como diferentes configuraciones de los mismos principios, lo cual hace pensar que ninguno puede verse como un constructo "puro" o independiente de todos los demás, por lo que en la búsqueda por la mejor forma de enseñar idiomas, hay quienes han pensado que es pertinente pensar fuera del los constructos de los métodos.

\section{La Condición Postmétodos}

Algunos autores han intentado referirse a lo que ha de esperarse si se reflexiona o se va más allá de los métodos para la enseñanza de idiomas. Richards y Rodgers (2004) sugieren que en la era postmétodos, el docente de idiomas debería buscar su propio enfoque para enseñar lenguas y brindan algunas pautas generales. Sin embargo, estas sugerencias no brindan herramientas e insumos suficientes para que un docente de idiomas realice el paso evolutivo trascendental más allá de los métodos. Por otro lado, Hall (2011) considera que la noción de un postmétodo no dista mucho de la de un método, presumiblemente porque piensa que un postmétodo también supondría prescripciones metodológicas y teóricas para los docentes de idiomas. A pesar de esto, lo que Hall (2011) hace básicamente es criticar ideas ajenas sobre la era postmétodos, pero no llega si quiera a proponer su propia perspectiva acerca del tema. No obstante, existe un autor, quien a través de los años, ha tratado de problematizar qué ruta podría seguirse al ir más allá de las fronteras de los métodos. Kumaravadivelu $(2008,2006,2003,1994)$ la ha denominado la "condición post métodos". La condición postmétodos no es un nuevo método para la enseñanza de idiomas; podría verse más como otra etapa en la evolución de esta disciplina y consiste en diez macroestrategias para la enseñanza de idiomas. A continuación, se 
describirán de manera general las diez macroestrategias ${ }^{2}$ para la enseñanza de idiomas propuestas por Kumaravadivelu (2008, 2003, 1994):

\section{Maximizar las oportunidades de aprendizaje}

Para promover un aprendizaje significativo, el docente de idiomas debe brindar a sus estudiantes abundantes insumos en el salón de clase. También, el docente debe procurar alejarse de lo magistral y permitir que las lecciones se centren más en el estudiante. Esto requiere, por lo tanto, flexibilidad en el planeamiento didáctico para conocer las necesidades del estudiantado y potenciar sus destrezas.

\section{Minimizar los errores de percepción}

Para lograr comunicarse asertivamente es necesario expresar mensajes claros. Esta debería ser una máxima para todo docente. Muchas veces existe una gran brecha entre lo que intenta explicar un docente y lo que en última instancia comprenden los estudiantes. En conclusión, los docentes de idiomas deben volverse mejores mediadores y monitorear constantemente la manera en que se expresan y dan instrucciones a sus estudiantes. Kumaravadivelu (2008) considera que en este sentido existen diez categorías de posible incertidumbre, a saber, 1. Cognoscitiva. 2. Comunicativa. 3. Lingüística. 4. Pedagógica. 5. Estratégica. 6. Cultural. 7. Evaluativa. 8. Procedimental. 9. Instruccional y 10 . Actitudinal.

\section{Facilitar la interacción negociada}

La comunicación como fenómeno social requiere que diversos individuos interactúen entre sí de una manera coherente. En la enseñanza de idiomas, esto debe promoverse de toda forma posible. No solo el docente debería interactuar con los estudiantes; estos deben también interactuar ente sí y con el docente. Es pertinente también, sugiere quien escribe, que los docentes vean los procesos de enseñanza y aprendizaje

2 Para un abordaje más profundo de cada una de las macr estrategias, se sugiere consultar la obra de Kumaravadivelu (2003). 
de una manera multidireccional. O sea, no solo el docente enseña y los estudiantes aprenden; los estudiantes también pueden enseñar a sus pares y al docente. Desde luego, para que la interacción negociada ocurra eficazmente, el docente debe ser un facilitador en este proceso.

\section{Promover la autonomía del aprendiz}

En el siglo XXI, los estudiantes no deben ser vistos ni verse a sí mismos como entes pasivos receptores de información. El aprendiz actual debe responsabilizarse por su propio aprendizaje, debe aprender a aprender (y a reaprender y desaprender si fuese necesario), debe conocerse a sí mismo en sus estilos y estrategias de aprendizaje y, sobre todo, debe tener hambre por aprender y no conformarse únicamente con lo que pueda recibir en un salón de clases. Para poder lograr esto, los docentes deben guiar y orientar a los estudiantes hacia buenas prácticas de estudio y de aprendizaje y hacia el autoconocimiento. Esto quiere decir que en la actualidad, ninguna persona será un producto terminado una vez que se gradúe; todos hemos de ser aprendices de por vida.

\section{Promover la consciencia lingüistica}

Para poder aprender y en última instancia utilizar de manera correcta un idioma, es necesario conocer como funciona ese sistema lingüístico. Se debe ayudar a los estudiantes a ver cuáles reglas o principios existen en una lengua. Se puede añadir también que, en el caso de la enseñanza de lenguas extranjeras, hay que guiar a los estudiantes para que noten qué similitudes y diferencias existen entre su lengua materna y la lengua que estén aprendiendo, para que así puedan comprender qué es posible y qué no en un idioma extranjero desde un punto de vista lingüístico.

\section{Activar la heurística intuitiva}

Naturalmente no es factible ni práctico aprender cada regla o principio existente para una determinada lengua. Es ahí donde entra la heurística intuitiva: la habilidad de inferir reglas y de generar significado por medio del contexto. Por esta razón, un aprendiz de idiomas eficiente y autónomo debe desarrollar sus destrezas inferenciales 
para descubrir todo aquello que no pudo aprender formalmente en un aula. Para abordar esta macroestrategia, el docente de idiomas deberá proveer al estudiante de abundantes textos escritos y orales y guiar al estudiante hacia el autodescubrimiento de reglas y significados. En este sentido, se puede ampliar que, el docente de idiomas no debe olvidar que no es más que un aprendiz más experimentado que sus estudiantes, por lo que es conveniente que reflexione sobre sus propias estrategias para lidiar con lo nuevo y lo desconocido, para así poder compartirlas con sus estudiantes y estos puedan beneficiarse de esta experiencia.

\section{Contextualizar la entrada lingüística}

Las palabras adquieren su verdadero significado cuando ocurren en un contexto determinado. En la enseñanza de idiomas del siglo XXI, el contexto es crucial. El docente de lenguas debe alejarse de las prácticas anticuadas de presentar oraciones descontextualizadas, cuyo único propósito es ilustrar una determinada estructura gramatical. Por lo tanto, esto se podría lograr por medio del uso de materiales auténticos (artículos de periódicos, películas, programas de televisión, entre otros) para ir más allá de los libros de texto, los cuales muchas veces presentan visiones artificiales de los idiomas.

\section{Integrar las destrezas lingüísticas}

Cada lenguaje puede verse en términos de macrodestrezas (habla, escucha, lectura y escritura) y microdestrezas (pronunciación y gramática, por ejemplo). A través de los años, la aparente practicidad de segregar las destrezas de un idioma ha predominado en el campo de la enseñanza de lenguas. Sin embargo, tal separación está en completa disonancia con la realidad. Por ejemplo, no se puede hablar sin escuchar también, así como resulta muy difícil escribir sin antes haber leído. Cada destreza lingüística es como una vía de información, por lo que mientras más vías se estimulen cada vez, el aprendizaje de los estudiantes será más profundo y memorable. En conclusión, para propiciar un mejor aprendizaje en los estudiantes, el docente de idiomas debe planear actividades de clase que integren tantas destrezas lingüísticas como sea posible. 


\section{Garantizar la relevancia social}

La naturaleza emancipadora de la educación no puede estar ausente en el campo de la enseñanza de idiomas. El docente de idiomas debe estar consciente de la realidad socioeconómica y sociopolítica en la cual desempeña su práctica educativa; debe empoderar al estudiante para que tenga una visión crítica de su realidad. Por ejemplo, en el pasado se creía que el objetivo último de aprender otro idioma era hablar y ser como un hablante nativo de ese idioma, lo cual en muchos casos provoca el desprecio por lo propio, lo criollo, lo autóctono. Un aprendiz de idiomas crítico y sensible de su realidad no debe olvidar de dónde viene y cuál es su identidad para que pueda utilizar dos (o más) idiomas exitosamente.

\section{Crear consciencia cultural}

En la actualidad, es necesario que los estudiantes desarrollen una consciencia global. Para lograrlo, se debe trabajar en desafiar los estereotipos culturales que existen, descubrir lo que cada cultura es realmente y promover la comprensión y la tolerancia entre los pueblos. Desde el punto de vista del campo de la comunicación intercultural, también conviene aprender sobre las costumbres extranjeras e identificar las similitudes y diferencias con las propias, ya que muchos malos entendidos surgen cuando se piensa que todas las culturas interactúan de una misma forma. La cultura es vista ahora como una destreza lingüística más, por lo cual para utilizar un idioma de manera competente, también se debe ser competente culturalmente (Celce-Murcia, 2007).

Es importante aclarar en este momento que, todas estas diez macroestrategias para la enseñanza de idiomas son únicamente propuestas generales de acción para el docente de idiomas. Kumaravadivelu (2008) considera cada una de las diez macroestrategias como neutras con respecto tanto a teorías del lenguaje, aprendizaje o enseñanza como a los principios metodológicos de los métodos para la enseñanza de idiomas. Por lo tanto, el docente de idiomas que desee utilizar las macroestrategias no tendrá que lidiar con imposiciones teóricas o metodológicas. El desafío que ha de enfrentar el docente de idiomas entonces será desarrollar sus propias microestrategias para la enseñanza de idiomas, las cuales sirvan para abordar las macroestrategias. La condición postmétodos 
es una propuesta sugerente e interesante. No obstante, el poder trabajar en este paradigma supone grandes retos.

\section{Desafíos que Afronta la Condición Postmétodos}

Para poder poner en práctica las ideas y macroestrategias de la condición postmétodos, es necesario franquear ciertos obstáculos. El primero de ellos es la formación docente (Akbari, 2008). Los cursos que aborden temas sobre metodologías para la enseñanza de idiomas deben ser replanteados para cambiar las visiones arraigadas de por qué se deben estudiar los diferentes métodos para la enseñanza de idiomas propuestos a través de la historia y evitar los malos entendidos del eclecticismo. Quizás por esta razón y razones políticas, sociales y económicas, se ha reportado que no es factible aplicar la pedagogía postmétodos (Khatib y Fathi, 2014; Khatib y Fat'hi, 2012). También, para poder formar docentes en la actualidad, el uso de libros de texto puede ser una limitante, ya que como lo considera McMorrow (2007), pueden favorecer prácticas neo-colonialistas por encima de los saberes y recursos locales. Otra área de crítica hacia la condición postmétodos es que, de acuerdo con Hashemi (2011), no es más que la propuesta de otro método para la enseñanza de idiomas o incluso una mera reinterpretación de la enseñanza comunicativa de idiomas (CLT, por sus siglas en inglés). Finalmente, un desafío más para la condición postmétodos es la cantidad de responsabilidad que recae sobre los docentes (Kamali, 2014), la cual se una a la ya muy larga lista de tareas en el quehacer de un docente de idiomas. En fin, todos estos retos pueden ser la razón por la cual en la actualidad se abusa de los recursos didácticos y de los libros de texto, tal como se explicará a continuación.

\section{Una Visión Diferente en una Era de Abundancia: Dogma}

En el siglo XXI, la cantidad de recursos didácticos existente puede ser abrumadora (libros, videos, grabaciones de audio, aplicaciones para dispositivos móviles, plataformas de aprendizaje en Internet, pizarras inteligentes, software interactivo, entre otros). Tal abundancia provoca que algunos docentes caigan en una especie de gula de recursos didácticos y crean que hay que usar estos insumos por el simple hecho de que están disponibles sin pensar en cuál valor añadido podrían 
brindar en los procesos de enseñanza y aprendizaje. Muchas veces el resultado obtenido son lecciones recargadas de materiales, los cuales no se logran explotar debidamente. En la era de la abundancia, Thornbury $(2005,2000)$ ha sugerido una propuesta minimalista, al pensar que las lecciones de los cursos de idiomas han sido secuestradas por tantos recursos didácticos distintos y por una obsesión compulsiva por lo gramatical. Antes de describir esta propuesta metodológica, es necesario aclarar, tal como el mismo Thornbury $(2010,2000)$ lo ha hecho, que su idea no es un nuevo método para la enseñanza de idiomas. Podría verse quizás como una filosofía o un paradigma más propiamente.

A Thornbury (2000), le llamó la atención que un grupo de cineastas daneses se agrupara e hiciera lo que el describe como un "voto de castidad" para librarse de lo artificial de los efectos especiales y escenografías creadas para las películas. Al analizar los postulados del grupo Dogme 95, Thornbury (2000) consideró que se podrían extrapolar a la enseñanza de idiomas para tratar de lograr más con menos recursos; de ahí Thornbury (2000) acunó el término "dogma." En la visión minimalista de Thornbury $(2005,2000)$, el docente y los estudiantes son los principales insumos para aprender un idioma. No quiere decir, supone quien escribe, que se deba prescindir de todo recurso publicado o producido para la enseñanza de idiomas, sino más bien que los recursos didácticos que se vayan a usar en clase deben ser útiles más allá de ser llamativos o nuevos, deben ser escogidos con cautela y criticidad y deben demostrar más allá de cualquier duda que producen mejores resultados que enfoques tradicionales.

En esta visión, todos los participantes del proceso de enseñanza y aprendizaje deben ser más autónomos y apropiarse de las lecciones del curso y ajustarlas a sus necesidades e intereses; el aprendizaje debe ser más experiencial y experimental. Así pues, el diálogo entre todos los participantes es primordial (Thornbury, 2005) para lograr los resultados deseados. En este sentido, Thornbury (2005) llama la atención al hecho de que "dogma" posee muchas similitudes con perspectivas como las de la educación humanista y la pedagogía crítica. Una vez más, nada de esto suena aparentemente innovador. No obstante, en la actualidad tal vez esto es lo que todos necesitamos. Las nuevas tecnologías facilitan nuestras vidas, son útiles, pero nos pueden alienar, nos pueden despojar de nuestra humanidad, de nuestra solidaridad y de nuestra criticidad. Lo que nos hace humanos no puede quedar de 
lado en ningún ámbito educativo. La educación nunca debería verse como algo mecánico ni genérico, en el cual los estudiantes pasan por un troquel que los moldea de un único modo. Lamentablemente, en la actualidad las casas editoriales en muchos casos privan a los docentes de libertades y flexibilidad pedagógicas.

\section{Una advertencia muy actual: la práctica definida por el libro de texto}

Hay muchos que ven la enseñanza como algo muy sencillo y desvirtúan el rol que puedan desempeñar los docentes en los procesos de enseñanza y aprendizaje. Por eso, se cree erróneamente que, existen fórmulas de enseñanza que cualquiera puede seguir y obtener los mismos resultados. Richards (2001, p.169) ha argumentado que una suposición detrás de la proposición de los métodos para la enseñanza de idiomas es que no se puede confiar en que los docentes enseñen bien. Por lo tanto, los docentes deben seguir recetas prescritas por "expertos" para no equivocarse en su práctica profesional. En esta misma línea de pensamiento, existe un fenómeno actual y aún más pernicioso: la práctica definida por el libro de texto (Akbari, 2008). Ahora es cada vez más común ver que en diferentes instituciones educativas, los libros se convierten en cursos o los cursos se convierten en libros (lo que suceda primero). Casas editoriales vienen a ofrecer sus series didácticas y estas se adoptan sin mayor criterio de selección; lo que sucede a continuación es que se toman los programas de curso y se sustituyen los contenidos por los que aparezcan en la tabla de contenidos de los libros. Quizás no exista nada más limitante para la labor docente que este fenómeno seudocurricular y colonizador, en el cual los docentes se ven forzados a seguir un libro de texto que ha sido prescrito por personas que probablemente no conocen los diseños curriculares existentes en un país o en un centro educativo, ni mucho menos los contextos socioeconómicos en los cuales se van a utilizar los libros.

Es preocupante también ver que muchos docentes vean a estos libros de texto como aliados, especialmente cuando son extensos, porque así pueden dedicarse a seguirlos en clase sin tener que planear lecciones ni preparar muchos (o ningunos) materiales didácticos. Grande es la queja por parte de estos docentes cuando en una institución o programa de idiomas se ponen en práctica libros "simples" o "pequeños", ya 
que esto los saca de su zona de confort y los obliga a complementar los libros con materiales adicionales. Es triste que no vean esto como una oportunidad. El que a un docente le den un libro simple o corto es el mayor respeto que le puedan brindar como profesional, porque así puede dedicar más tiempo y esfuerzo a atender las necesidades reales de sus estudiantes. Los libros de texto son propuestas genéricas, globales e idealizadas. Un docente crítico debería estar consciente de que su desafío radica en tomar esa propuesta, adaptarla y volverla algo local, específico y emancipador.

\section{Conclusión}

El campo de la enseñanza de idiomas tiene una larga (pero no necesariamente innovadora) historia y, como se ha descrito en este ensayo, su desarrollo no ha sido necesariamente evolutivo, yendo desde la nada hacia el algo. Esto quiere decir que, en cursos sobre métodos para la enseñanza de idiomas, aunque se trate de trazar una línea histórica del método más antiguo al más moderno, el progreso de la disciplina no es lineal, sino más bien ha sido una espiral de contante reconfiguración. En este sentido, como lo sugiere el título de este escrito, no hay nada nuevo bajo el sol. Es posible identificar nueve espectros de posibilidades metodológicas en prácticamente todos los métodos para la enseñanza de idiomas. Aún así, conocer los alcances de cada una de estas dimensiones podría brindar insumos para el planeamiento de lecciones.

Por otro lado, la búsqueda del mejor método para la enseñanza de idiomas puede convertirse en un esfuerzo infructuoso hasta que cada docente se dé cuenta de que lo que realmente cuenta es el compromiso y el esfuerzo que se le pongan al quehacer diario en el aula. De ahí es que quizás surgen propuestas como la condición postmétodos y dogma, las cuales, a pesar de sus desafíos, buscan empoderar al docente de idiomas en su práctica profesional. Finalmente, se ha querido llamar la atención hacia el camino menos alentador de la enseñanza de idiomas: la práctica definida por el libro de texto y sus implicaciones.

En este momento postmoderno de la enseñanza de idiomas, queda a criterio de cada docente reflexionar sobre el camino seguido en su práctica y decidir si conviene moverse hacia delante o si vale la pena cuestionar críticamente lo hecho hasta el momento y re-crearse como profesional. En el siglo XXI, ningún docente debe dejar de aprender y 
debería investigar y reflexionar sobre su quehacer académico para realizar cambios que beneficien al estudiantado y, en última instancia, al desarrollo social. Estas decisiones supondrán en muchos casos luchar contra sistemas educativos y políticos ineficientes y lidiar con diversas limitaciones. Será cuestión de cada uno aceptar el desafío o no.

\section{Referencias bibliográficas}

Akbari, R. (2008). Postmethod discourse and practice. TESOL Quarter$l y, 42$ (4), 641-652.

Celce-Murcia, M. (2007). Rethinking the role of communicative competence in language teaching. En E. Alcón y M. P. Safont (Eds.), Intercultural Language Use and Language Learning (pp. 41-57). Dordrecht: Springer.

Hall, G. (2011). Exploring English language teaching: Language in action. New York: Routledge.

Hashemi, S. (2011). (Post)-Methodism: Possibility of the impossible? Journal of Language Teaching and Research, 2 (1), 137-145. doi:10.4304/jltr.2.1.137-145

Kamali, J. (2014). Postmethod survival. Procedia-Social and Behavioral Sciences, 98, 824-829.

Khatib, M. y Fathi, J. (2014). The investigation of the perspective of Iranian EFL domain experts on post-method pedagogy: A delphi technique. The Journal of Teaching Language Skills, 6 (3), 101-124.

Khatib, M. y Fat'hi, J. (2012). Postmethod pedagogy and ELT teachers. Journal of Academic and Applied Studies, 2 (2), 22-29.

Kumaravadivelu, B. (2008). Understanding Language Teaching: From Method to Postmethod. New Jersey: Lawrence Erlbaum Associates.

Kumaravadivelu, B. (2006). TESOL methods: Changing tracks, challenging trends. TESOL Quarterly, 40 (1), 59-81.

Kumaravadivelu, B. (2003). Beyond methods: Macrostrategies for language teaching. New Haven: Yale University Press.

Kumaravadivelu, B. (1994). The post method condition: (E)merging strategies for second/foreign language teaching. TESOL Quarterly, 28 (1), 27-48. 
McMorrow, M. (2007). Teacher education in the postmethods era. ELT Journal, 61 (4), 375-377.

Prabhu, N. S. (1990). There is no best method-Why? TESOL Quarter$l y, 24(2), 161-176$.

Richards, J. C. (2001). Beyond methods. En C. N. Candlin y N. Mercer (Eds.), English language teaching in its social context: A reader (pp. 167-179). New York: Routlege.

Richards, J. C. y Rodgers, T. (2004). Approaches and methods in language teaching $\left(2^{\text {nd }} \mathrm{Ed}\right.$.). New York: Cambridge University Press.

Thornbury, S. (2010). The secret history of methods. Recuperado de https://www.youtube.com/watch?v=L2q9B2BEV2U

Thorrnbury, S. (2005). Dogme: Dancing in the dark. Folio 9 (2), 3-5.

Thorbury, S. (2000). A dogma for EFL. IATEFL Issues, 153, 2. 